\title{
Coherent Transition Radiation From a Laser Wakefield Accelerator as an Electron Bunch Diagnostic
}

\author{
J. van Tilborg*†, C. G. R. Geddes ${ }^{* * *}$, C. Tóth*, E. Esarey*, \\ C. B. Schroeder*, M. C. Martin*, Z. Hao* and W. P. Leemans* \\ ${ }^{*}$ Lawrence Berkeley National Laboratory, University of California, Berkeley, CA 94720 \\ ${ }^{\dagger}$ Technische Universiteit Eindhoven, the Netherlands \\ ${ }^{* *}$ Department of Physics, University of California, Berkeley, CA
}

\begin{abstract}
The observation and modeling of coherent transition radiation from femtosecond laseraccelerated electron bunches is discussed. The coherent transition radiation, scaling quadratically with bunch charge, is generated as the electrons transit the plasma-vacuum boundary. Due to the limited transverse radius of the plasma boundary, diffraction effects will strongly modify the angular distribution and the total energy radiated is reduced compared to an infinite transverse boundary. The multi-nC electron bunches, concentrated in a length of a few plasma periods (several tens of microns), experience partial charge neutralization while propagating inside the plasma towards the boundary. This reduces the space-charge blowout of the beam, allowing for coherent radiation at relatively high frequencies (several THz). The charge distribution of the electron bunch at the plasma-vacuum boundary can be derived from Fourier analysis of the coherent part of the transition radiation spectrum. A Michelson interferometer was used to measure the coherent spectrum, and electron bunches with duration on the order of $50 \mathrm{fs}(\mathrm{rms})$ were observed.
\end{abstract}

\section{INTRODUCTION}

Ever since the introduction of particle accelerators, the need for beam characterization has been present. Beam properties, such as electron bunch charge, electron energy, beam divergence, transverse and longitudinal charge distribution, yield relevant information on the performance of the accelerator.

One group of characterization techniques relies on the emission of electromagnetic radiation by the electron beam during interaction with various dielectric structures. Cherenkov radiation [1], emitted when a bunch passes through a medium where the electron velocity exceeds the local phase velocity of the electromagnetic wave, diffraction radiation [2], and transition radiation [3-5], generated as a bunch passes through a change in dielectric structure (transverse change or longitudinal change, respectively), are examples of emission processes that can be used to characterize the beam.

The emitted radiation can be incoherent or coherent radiation. As a rule of thumb, radiation at wavelengths shorter than the longitudinal bunch duration is incoherent (proportional to the number of electrons in the bunch), while radiation at longer wavelengths is coherent, in which case the radiation scales as the square of the number of electrons. For typical electron beams in conventional accelerators (as well as novel laser-based ac- 
celerators), with $10^{6}-10^{10}$ electrons per bunch, the spectrum of the radiation is fully dominated by the coherent part due to this quadratic dependence. The coherent radiation represents the Fourier transform of the charge distribution.

In this work, the characterization of coherent transition radiation generated by relativistic electrons, produced in a laser-plasma accelerator, is discussed. The concept of an ultra-intense laser pulse driving a charge density oscillation in a plasma, with longitudinal electric fields large enough to accelerate electrons to relativistic velocities, was predicted by Tajima and Dawson in 1979 [6] and has been observed in several labs worldwide in the past decade [7, 8]. The introduction of the Chirped Pulse Amplification technique was crucial to realizing the level of laser intensity [9] needed for this non-linear plasma response. The accelerator, also referred to as LWFA (laser wakefield accelerator), can either accelerate externally injected electrons, or rely on the self-trapping of background plasma electrons. The experiment discussed in this work has been performed at the Lawrence Berkeley National Laboratory (LBNL) using a single-beam configuration, i.e., a single laser beam is used to ionize the gas, generate the plasma density oscillation, and trap background plasma electrons.

The accelerator performance of such a single-beam LWFA at LBNL has been discussed by Leemans et al. [4, 10]. It is important to note that the electron beam properties change once the electron beam exits the plasma, since the high charge density induces a space-charge blow-out [11]. In order to characterize this accelerator at or nearby the source (i.e., the plasma), use of a radiation mechanism before space-charge blow-out is essential. Such a mechanism exists, since the plasma-vacuum boundary represents a longitudinal change in dielectric function, which produces transition radiation $[4,5,12]$. The radiation from this boundary is analyzed, yielding information about the charge distribution before space-charge forces act on the electrons. The detector used is a Michelson interferometer. The interferogram is Fourier transformed, and the obtained coherent spectrum is compared to results from an analytical model.

In the following two sections a short theoretical background to transition radiation is given, as well as the presentation and discussion of the experimental setup, results and, analysis.

\section{TRANSITION RADIATION FROM A FINITE BOUNDARY}

The theory of transition radiation by a single electron, first studied by Ginzburg and Frank [13], is extensively treated in the monograph by Ter-Mikaelian [14]. Transition radiation produced by laser-plasma generated electron bunches transiting a plasmavacuum interface was studied by Schroeder et al. [5].

In the following analytical analysis, it is assumed that the coherent radiation spectrum is dominated by the longitudinal charge distribution, and not the transverse charge distribution. The bunch length is expected to be several plasma periods $\lambda_{p}$ long $\left(\lambda_{p} \simeq\right.$ $6 \mu \mathrm{m}$ ), while the transverse size of the bunch is predicted to be on the order of the laser spotsize $w_{0}$, with $w_{0} \simeq 6 \mu \mathrm{m}$ [15]. Therefore, the longitudinal charge distribution will determine the spectral width of the coherent transition radiation.

The dielectric function $\varepsilon(\omega)$ changes from $\varepsilon_{1}(\omega)=1-\omega_{p}^{2} / \omega^{2}$ in the plasma to 
$\varepsilon_{2}(\omega)=1$ in the vacuum, with $\omega_{p}=2 \pi c / \lambda_{p}$. Since, for the coherent frequencies, $\omega_{p}^{2} / \omega^{2} \gg 1$, one can approximate $\varepsilon_{1}(\omega)$ by $\varepsilon_{1}(\omega)=-\infty$, i.e., we may treat the boundary approximately as a conductor-vacuum interface, which simplifies the analysis.

Based on interferometric measurements on the density profile $n(r, z)$, the density gradient at the boundary occurs on a shorter scale than the formation length $L_{T R}$ of the transition radiation [16]. The formation length can be expressed as $L_{T R}=\lambda /\left(\theta^{2}+\gamma^{-2}\right)$, where $\theta$ represents the angle of observation, and $\gamma$ the relativistic factor of the electrons. In the experiments discussed here, $\theta \lesssim 0.15 \mathrm{rad}$, and $\gamma \simeq 6$. For coherent wavelengths around $\lambda \simeq 100 \mu \mathrm{m}$, the formation length is several millimeters long, while the plasma boundary has a width on the order of several hundred micron. Therefore the plasmavacuum interface may be treated as a sharp boundary.

With the above assumptions and considering an electron beam traveling normal to the boundary (and neglecting divergence effects), the differential energy spectrum of the coherent transition radiation can be written as [5]

$$
\frac{d^{2} W}{d \omega d \Omega}=\frac{e^{2}}{\pi^{2} c} N^{2}|\langle\mathcal{E} D F\rangle|^{2}
$$

where $d^{2} W / d \omega d \Omega$ is the energy radiated per unit of frequency bandwidth $d \omega$, and per unit of solid angle $d \Omega$. The brackets denote averaging over electron energy distribution. Electron energy measurements on beams from LWFA's have shown a typical Boltzmann like energy distribution, with a temperature on the order of 3-4 MeV. The number of electrons in the bunch is $N$ (note the coherent $N^{2}$ dependence). Within the brackets the three functions $\mathcal{E}, D$ and $F$ are introduced: $\mathcal{E}$ represents the angular distribution function, including the electron energy dependence, given by

$$
\mathcal{E}(\theta, u)=\frac{u \sqrt{1+u^{2}} \sin \theta}{1+u^{2} \sin ^{2} \theta},
$$

where $u=\gamma v_{z} / c$ ( $v_{z}$ is the velocity in the direction normal the boundary) and $\theta$ is the angle of observation, measured with respect to the $z$-axis. The function $D$ represents the diffraction function, since the interface has a finite transverse dimension $\rho$. For long wavelengths $(2 \pi \rho / \lambda u<1)$, diffraction effects are important, and $D \rightarrow 0$. The diffraction function is given by

$$
D(b, u \sin \theta)=1-J_{0}(b u \sin \theta)\left[b K_{1}(b)+\frac{b^{2}}{2} K_{0}(b)\right]-\frac{b^{2}}{2} K_{0}(b) J_{2}(b u \sin \theta) .
$$

Here $J_{m}$ and $K_{m}$ are the $m^{\text {th }}$ order regular and modified Bessel functions, respectively, and the dimensionless impact parameter $b$ is given by $b=k \rho / u$, where $k=2 \pi / \lambda$. Since $\rho$ is on the order of several hundred micron, $b$ is of order unity, indicating diffraction strongly affects the spectral and angular energy distribution. Finally, the function $F$, or the formfactor, represents the degree of coherence and is simply given by the Fourier transform of the longitudinal charge distribution. For a Gaussian charge distribution, with rms length given by $\sigma_{z}$,

$$
F\left(\omega, v_{z}\right)=\exp \left[-\left(\omega \sigma_{z} / v_{z}\right)^{2} / 2\right]
$$




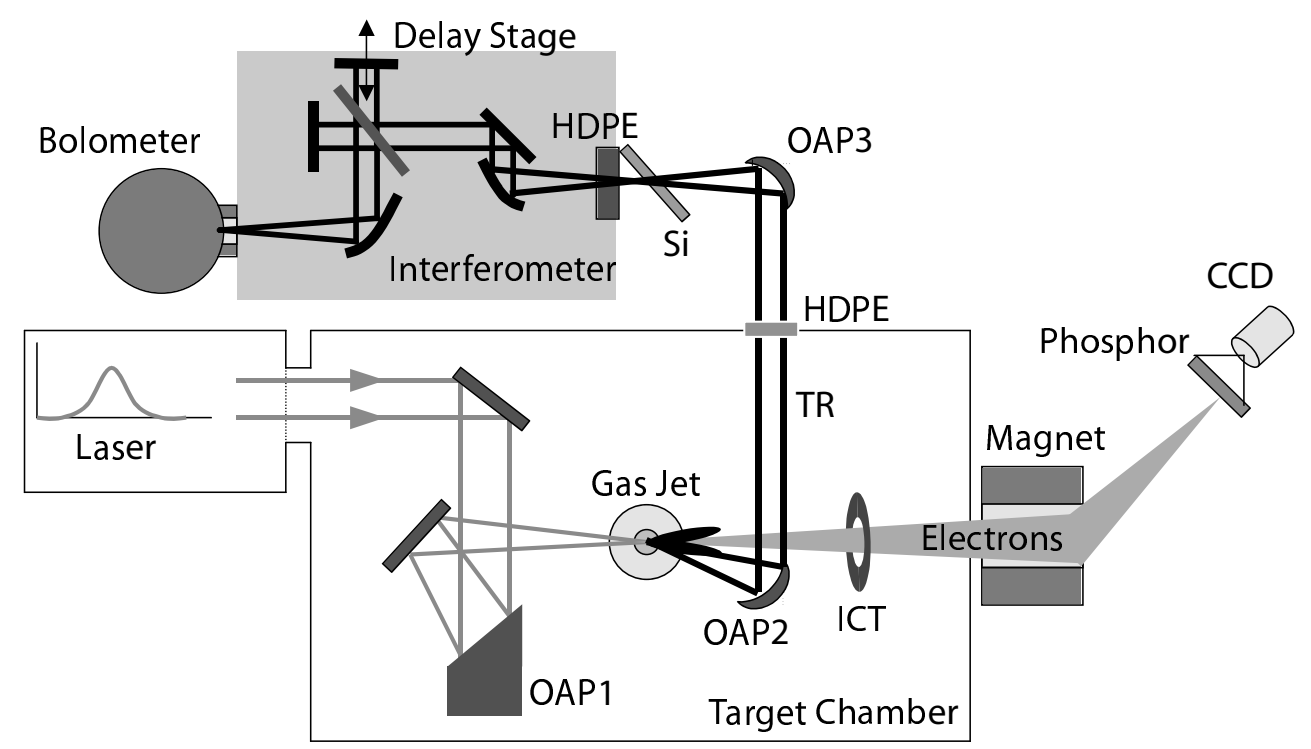

FIGURE 1. Schematic of the target area of the L'OASIS laser system. The transition radiation (TR) is coupled into the Michelson Interferometer through OAP2 and OAP3. It propagates through two HighDensity PolyEthylene windows (HDPE), as well as a Silicon wafer.

\section{EXPERIMENTAL SETUP AND RESULTS}

The experiments described in this paper were performed with the $10 \mathrm{~Hz} \mathrm{Ti}: \mathrm{Al}_{2} \mathrm{O}_{3} \mathrm{CPA}$ laser system at the L'OASIS Lab [17] at LBNL. A schematic of the setup in and around the target chamber is given in Figure 1. Several amplification stages produce a $50 \mathrm{fs,}$ $0.5 \mathrm{~J}$ laser pulse on target. Since the beam is focused using a F/4 off-axis parabola (OAP1), resulting in a spot on the order of $6 \mu \mathrm{m}$ (FWHM), the peak laser intensity reaches values of $1-2 \cdot 10^{19} \mathrm{Wcm}^{-2}$. The target is Helium gas, emerging from a circular nozzle (diameter of $2 \mathrm{~mm}$ ), producing a plasma with a typical length of $2 \mathrm{~mm}$ and width of several hundred micron. The plasma density is $n \simeq 3 \cdot 10^{19} \mathrm{~cm}^{-3}$, corresponding to a plasma wavelength of $\lambda_{p}=6 \mu \mathrm{m}$.

The electron beam, generated in this small volume of plasma, will exit the plasma and pass through a Integrated Current Transformer (ICT) to measure the total bunch charge. The current through the magnet can be scanned to provide an energy spectrum of the electrons (for a given magnet current, electrons within a specific energy range are focused onto a phosphor screen, which emits light onto a CCD camera). Previous work on these diagnostics $[4,10]$ have indicated that multi-nC electron bunches are created, with a typical energy spectrum that is Boltzmann-like (temperature around 3-4 MeV).

In order to collect the transition radiation (TR), one would ideally install a parabola along the $z$-axis (laser propagation axis) for optimum and symmetric collection. However, a significant portion of laser beam escapes the plasma, and interaction with any solid object close to the target will result in damage of optics. Therefore an off-axis parabola (OAP2), with a diameter of $76.2 \mathrm{~mm}$, and an effective focal length of 152.4 $\mathrm{mm}$, is placed at a position where the $800 \mathrm{~nm}$ laser beam will not interact with the sur- 
(a)

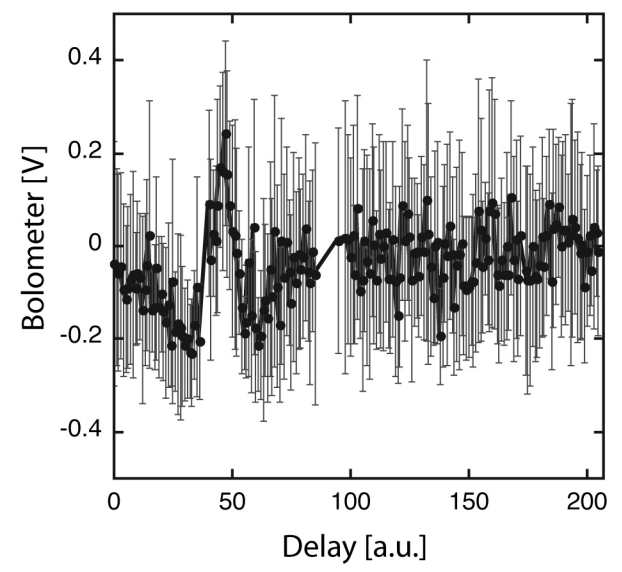

(b)

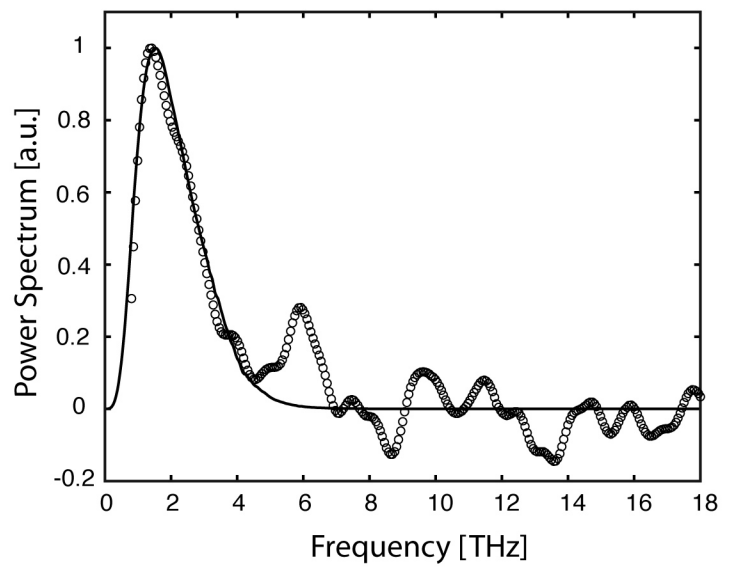

FIGURE 2. (a) The measured interferogram of the transition radiation. Each data point represents an average of 15 shots, and the standard deviation of these shots is indicated by the error bars. (b) The Fourier transform of the interferogram, representing the coherent power spectrum. The solid line is a best fit based on theory, after varying the parameters for the transverse plasma size $\rho$ and the electron bunch length $\sigma_{z}$.

face (an angle of 0.16 rad off the main $z$-axis). The aluminum parabola is uncoated, and collimates the TR. The TR is coupled out of the chamber through a $3.2 \mathrm{~mm}$ thick HighDensity PolyEthylene (HDPE) window. A high-resistivity Silicon wafer is installed to fully block out any remnant $800 \mathrm{~nm}$ laser light. A third off-axis parabola, the 90 -degree OAP3 with an effective focal length of $177.7 \mathrm{~mm}$, is used to focus the TR into the Bruker IFS66v/S Michelson Interferometer, through a second HDPE window. The beamsplitter in the interferometer is a $6 \mu \mathrm{m}$ thick Mylar splitter. Both the target chamber as well as the Michelson Interferometer were pumped out to vacuum. The detector used for the measurements was a Silicon composite bolometer, manufactured by Infrared Laboratories, sensitive in the $0.3-30 \mathrm{THz}$ range. The spectral transmission curves for the HDPE window, the Silicon wafer, as well as the spectral response of the Interferometer have been experimentally determined in previous, unpublished work.

To obtain the interferogram, the delay stage in the interferometer was scanned over 212 positions. At each position, bolometer signals for 15 shots were saved by the integrated data acquisition/control system. The repetition rate of the laser during this specific experiment was $2 \mathrm{~Hz}$. The interferogram that was measured is plotted in Figure 2(a). The error bars represent the standard deviation given by the averaging at each specific position of the delay arm in the interferometer. The signal-to-noise ratio is very poor, on the order of 2-3, since the shot-to-shot reproducibility of the LWFA in the singlebeam regime needs significant improvement. The low reproducibility is a combination of various factors, such as the variation in laser pulse length, laser energy, laser pointing, plasma parameters, seed for the numerous nonlinear laser/plasma interactions such as self-modulation and self-trapping, etc.. Future research will focus on single-shot characterization techniques.

It is known that a Fourier transformation of an interferogram is proportional to the 
power spectrum of the radiation. The second plot shows the obtained spectrum. The software package (OPUS) that comes with the Bruker Interferometer has a certain set of functions that perform data-smoothening of the raw interferogram before applying the Fourier transform. It is for this reason that the spectrum appears more smooth.

The black solid line shows a best fit using the freedom to vary two parameters. One is the transverse size of the plasma $\rho$, and the other one is the rms longitudinal bunch length $\sigma_{z}$. The effects of the transmission curves of the various optical components, as well as the spectral response of the interferometer are included in this best fit. It is found that the best fit is achieved by setting $\rho=500 \mu \mathrm{m}$, and $\sigma_{z}=15 \mu \mathrm{m}$ (or $50 \mathrm{fs}$ ). The plasma size corresponds to the line of expectation, although the actual size was never measured. This is because the density-interferometry experiments only image the size of the high density plasma $\left(>10^{18} \mathrm{~cm}^{-3}\right)$, and for the generation of TR it is the region of lower densities around $1 \cdot 10^{17} \mathrm{~cm}^{-3}$ that is affecting the radiation mechanism.

Previous measurements with the L'OASIS system [12] estimated the transverse plasma size on the order of $100 \mu \mathrm{m}$. It is not clear where the discrepancy between that number (derived from comparison of measured and calculated total energy) and this work's $500 \mu \mathrm{m}$ (derived from spectral characterization) originates. One possibility is that due to the longitudinal and transverse density distribution, the plasma acts as a negative lens for the $\mathrm{THz}$ radiation. The transverse plasma size may therefore indeed be on the order of $500 \mu \mathrm{m}$, and it is the smaller effective collection angle (due to the lensing) that brings down the total measured energy in [12]. Note that the spectrum of the TR is not very sensitive to the effective emission angle, validating the conclusions based in this proceeding. Due to various geometrical apertures in the TR beam line, only radiation close to the 0.16 rad emission angle makes it into the interferometer. Therefore the fit is based on the 0.16 emission angle, and a more complicated angular integration of the radiation is not found necessary.

Simulations have indicated that the electron bunch produced in a LWFA, or selfmodulated LWFA, has a length of several plasma wavelengths [15]. With a plasma wavelength around $\lambda_{p}=6 \mu \mathrm{m}$, the rms value of $\sigma_{z}=15 \mu \mathrm{m}$ seems to confirm this prediction.

Since the interferogram was constructed using many laser shots, the data represents an averaged interferogram, where unstable features are washed out. Furthermore, it is important to note that due to the limited bandwidth of this detection mechanism (Mylar beamsplitter, several windows, bolometer response, etc.), information on spectral components above $25 \mathrm{THz}$ is lost. Therefore it is not possible with the current setup to derive bunch substructure at the scale length of the plasma wavelength $\lambda_{p}$.

\section{SUMMARY}

The theory behind the generation of transition radiation from a finite boundary is introduced, and a spectral measurement of such radiation from a laser-wakefield accelerator is presented. Short electron bunches, with a rms length of $\sigma_{z} \simeq 15 \mu \mathrm{m}$ are inferred from spectral measurements. Experimental characterization is achieved through use of Michelson interferometry. The obtained spectrum reflects an averaged bunch length, 
since the measurement technique relies on the step-scan method. The experimental findings confirm theoretical predictions.

\section{ACKNOWLEDGMENTS}

This work has been supported by U.S. Department of Energy under contract No DEAC-03-76SF0098. C. G. R. Geddes acknowledges support from the Hertz Foundation.

\section{REFERENCES}

1. M. Uesaka et al., Nucl. Instr. and Meth. A 345, 219 (1994).

2. N. Potylitsina-Kube et al., Nucl. Instr. and Meth. B 201, 172 (2003).

3. Y. Shibata et al., Phys. Rev. E 50, 021479 (1994).

4. W. P. Leemans et al., Phys. Rev. Lett. 91, 074802 (2003).

5. C. B. Schroeder et al., Phys. Rev. E 69, 016501 (2004).

6. T. Tajima and J. Dawson, Phys. Rev. Lett. 43, 040267 (1979).

7. E. Esarey et al., IEEE Trans. Plasma Sci. 24, 252 (1996).

8. W. P. Leemans et al., these proceedings.

9. M. D. Perry and G. Mourou, Science 264, 917 (1994).

10. W. P. Leemans et al., Phys. Rev. Lett. 89, 174802 (2002).

11. G. Fubiani et al., Proceedings of the 2002 Advanced Accelerator Concepts Workshop, ed. C. Clayton and P. Muggli (AIP, 2002), Vol. 647, 203-212. 647, 203-212 (2002).

12. W. P. Leemans et al., Phys. Plasmas 11, 2899 (2004).

13. V. L. Ginzburg and I. M. Frank, Sov. Phys. JETP 16, 15 (1946).

14. M. L. Ter-Mikaelian, High-Energy Electromagnetic Processes in Condensed Media (WileyInterscience, New-York, 1972).

15. E. Esarey et al., Phys. Rev. Lett. 80, 255552 (1998).

16. J. V. Lepore et al., Phys. Rev. D 13, 082300 (1976).

17. C. Tóth et al., these proceedings. 